It had been pointed out earlier ${ }^{7}$ that the value of the intercept in a plot $I^{\mathrm{s}}\left(q_{0}\right)$ vs $\ln \left(q_{0}\right)$ was related to the size of the loops. In the more complete development presented in this paper it was shown that this intercept is at $q_{0}=e^{1 / 2} / R$ for defects of uniform radius $R$ and at $q_{0} \cong 1 / R_{0} e$ for an exponential size distribution. Hence the integral diffuse scattering measurements can be used to obtain quantitative measurements of loop sizes. From the electron

1 J. R. Patel and B. W. Battermann, J. Appl. Phys. 34, 2716 [1963].

2 O. N. Efimov and A. M. Elistratov, Soviet Phys. - Solid State 5, 1364, 1543 [1964] ; H. F. Wenzl, Z. Naturforsch. 26 a, 495 [1971].

${ }_{3}$ T. O. Baldwin, F. A. Sherrill, and F. W. Young, Jr., J. Appl. Phys. 39, 1541 [1968].

${ }^{4}$ L. S. Edelheit, J. C. North, J. G. Ring, J. S. Koehler, and F. W. Young, Jr., Phys. Rev. B 2, 2913 [1970].

${ }^{5}$ P. H. Dederichs, Phys. Rev. B 1, 1306 [1970]. microscopy studies, which indicate an exponential distribution, values for $R_{0}$ were obtained which are in approximate agreement with the values deduced from the present studies, Table 1 .

\section{Acknowledgement}

We thank T. H. Blewitt for performing the low temperature irradiations.

6 B. C. Larson and F. W. Young, Jr., Phys. Rev. B 4, 1709 [1971].

7 J. E. Thomas, T. O. Baldwin, and P. H. Dederichs, Phys. Rev. B 3, 1167 [1971].

${ }^{8}$ P. Dederichs, J. Physics, F (in press, 1973).

9 P. H. Dederichs, Phys. Rev. B 4, 1041 [1971].

10 D. B. deHaan, Nouvelles Tables d'Intégrales Définies, Hafner Publ. Co., New York 1957.

11 M. Rühle, F. Häussermann, and M. Rapp, Phys. Stat. Sol. 39, 609 [1970].

\title{
Use of Asymmetric Dynamical Diffraction of X-rays for Multiple-Crystal Arrangements of the $\left(\mathrm{n}_{1},+\mathrm{n}_{2}\right)$ Setting
}

\author{
Kan Nakayama *, Hiroo Hashizume, Akio Miyoshi, Seishi Kikuta **, and Kazutake Kohra \\ Department of Applied Physics, Faculty of Engineering, University of Tokyo, \\ Hongo, Bunkyo-ku, Tokyo \\ (Z. Naturforsch. 28 a, 632-638 [1973] ; received 1 February 1973) \\ Dedicated to Prof. Dr. G. Borrmann on the occasion of his 65 th birthday
}

\begin{abstract}
Asymmetric dynamical diffractions of X-rays are successively used for multiple crystals arranged in the $\left(n_{1},+n_{2}\right)$ setting. Double successive asymetric diffractions of the $\left(n_{1},+n_{2}\right)$ arrangement give a beam of peculiar spread in angle and wavelength. Triple successive asymmetric diffractions of the $(n,+n,-n)$ arrangement give a highly parallel and monochromatic beam for some appropriate combination of the asymmetry factors of the three crystals. Possible use of multiple asymmetric diffractions is discussed.
\end{abstract}

\section{Introduction}

Detailed information about dynamical diffraction phenomena of X-rays is obtained by using an incident beam of sufficiently narrow spread in angle and wavelength compared with the diffraction range of the orystal under investigation, which is of the order of seconds of arc. For such studies two kinds of arrangements are usually used: the double-crystal one of parallel or $(n,-n)$ setting and the triplecrystal one of $\left(n_{1},+n_{2}, \pm n_{3}\right)$ setting.

\footnotetext{
* Present address: National Research Laboratory of Metrology, Kaga, Itabashi-ku, Tokyo.
}

In the former the spread of the beam used is narrow in angle only for each wavelength component, but, as a whole, not very narrow in angle nor wavelength. However, the dispersion effect due to the wavelength spread is eliminated owing to the parallel setting, in which the interplanar spacings of the diffracting planes of the first and second (specimen) crystals are equal to each other. In the parallel setting an extremely narrow angular spread of the incident beam is obtained by making use of the effects characteristic to dynamical diffraction

\footnotetext{
** Present address: The Institute of Industrial Science, University of Tokyo, Roppongi, Minato-ku, Tokyo. Reprint requests to Dr. K. Kohra, University of Tokyo, Department of Applied Physics, Faculty of Engineering, Bunkyo-ku, Tokyo 113, Japan.
} 
such as Borrmann fan *,1-5, Borrmann absorption ${ }^{*}, 1,5$ and asymmetric diffraction 6,7 .

Recently the asymmetric diffraction has intensively been utilized for studying various dynamical diffraction phenomena such as asymmetric profiles $^{8-11}$ and subsidiary maxima ${ }^{12,13}$ of the diffraction curves from thick and thin crystals, respectively, accurate determination of $f$-values ${ }^{11,14,15}$ and Pendellösung interference fringes ${ }^{16,17}$. In these studies double or triple successive asymmetric diffractions arranged in the parallel setting have successfully been used in place of a single asymmetric diffraction in the first crystal.

On the other hand, in the triple-crystal arrangement ${ }^{18-21}$, an X-ray beam of sufficiently narrow spread in angle as well as wavelength is obtained from two crystals arranged in $\left(n_{1},+n_{2}\right)$ setting. Such a beam enables us to study any diffracting plane of any kind of crystal although the intensity is considerably weaker than that obtained in the double-crystal arrangement. Furthermore it would be possible, in principle, to study even minute changes of wavelength on inelastic scattering. In the triple-crystal arrangement some higher-order "symmetric" diffractions are used for the first and second crystals to make the angular and spectral spreads of the beam sufficiently narrower than the diffraction range of the third or specimen crystal.

In this paper we report on the use of asymmetric diffractions for the first and second crystals in the triple-crystal arrangement. It will be shown in Sect. 2 that, against expectation, the use of a single asymmetric diffraction for each of the two crystals does not improve the parallelism nor monochromacy of the obtained beam but gives us a peculiar spread in angle and wavelength of the beam. In Sect. 3 a method will be desribed to obtain a highly parallel and monochromatic beam by using triple successive asymmetric diffractions in the $(n,+n,-n)$ arrangement. In Sect. 4 possible use of multiple successive asymmetric diffractions will be discussed.

\section{The Nature of an X-ray Beam Obtained from Double Successive Asymmetric Diffractions in the $\left(n_{1},+n_{2}\right)$ Arrangement}

\subsection{Considerations in terms of the DuMond diagram}

The angular and wavelength spreads, $\theta$ - and $\lambda$-spreads, of $\mathrm{X}$-rays successively diffracted by two crystals are most easily known in terms of the Du-
Mond diagram ${ }^{22}$. We assume here the effects of the vertical divergence, i.e. the one perpendicular to the dispersing plane, of the X-ray beam to be negligibly small. Let us first consider the case where two crystals $\mathrm{C}_{1}$ and $\mathrm{C}_{2}$ are arranged in the symmetric $\left(n_{1},+n_{2}\right)$ setting (Figure 1). In this case the regions of selective diffraction for $\mathrm{C}_{1}$ and $\mathrm{C}_{2}$ are represented by bands $O_{1}$ and $O_{2}$, respectively, for both of incidence and emergence. The horizontal width of each band corresponds to the angular range of total reflection for a given wavelength for each crystal. The widths $\omega_{\mathrm{s} 1}$ and $\omega_{\mathrm{s} 2}$ are usually of the order of seconds of arc, but in Fig. 1 are drawn exaggerately for clarity. $\mathrm{X}$-rays successively diffracted by $C_{1}$ and $C_{2}$ are represented by the overlapped region of bands $O_{1}$ and $O_{2}$.

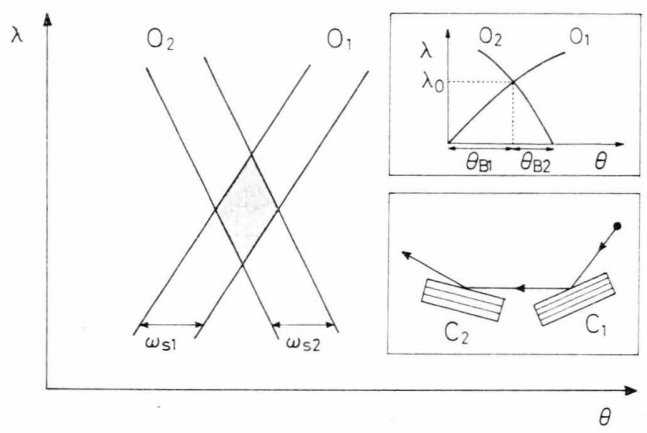

Fig. 1. DuMond diagram for two crystals $C_{1}$ and $C_{2}$ for the symmetric diffraction of the $\left(n_{1},+n_{2}\right)$ setting. An X-ray beam represented by the shaded region is obtained.

Before proceeding to the case where two crystals are arranged in the asymmetric $\left(n_{1},+n_{2}\right)$ setting, we consider the function of a single asymmetric diffraction in terms of the DuMond diagram (Figure 2). For an asymmetric diffraction, the angular ranges of total reflection for incidence and emergence for a given wavelength are given as $\omega_{0}=\omega_{\mathrm{s}} / V b$ and $\omega_{\mathrm{h}}=$ $\omega_{\mathrm{s}} \sqrt{b}$ respectively ${ }^{6,7}$, where $\omega_{\mathrm{s}}$ is the angular range of total reflection for the symmetric diffraction. $b$ is the asymmetry factor given by $b=\sin \left(\theta_{\mathrm{B}}-\alpha\right) /$ $\sin \left(\theta_{\mathrm{B}}+\alpha\right), \alpha$ being the angle between the diffracting plane and the crystal surface. $\alpha$ is taken positive when the incident beam makes an angle

\footnotetext{
* The use of the Borrmann fan was pointed out by Professor Borrmann himself to one of the authors (K. Kohra) as early as in 1955 and the use of the Borrmann fan as well as Borrmann absorption was made in 1957 by them at the Fritz-Haber Institute, Berlin, to measure rocking curves close to the intrinsic ones for calcite crystals. Some comments on the use of these two effects will be given in another place.
} 


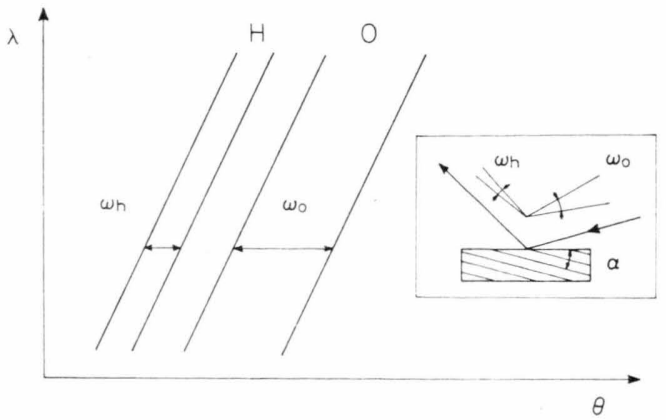

Fig. 2. DuMond diagram for an asymmetric diffraction. Diffraction regions for incidence and emergence are represented by bands $O$ and $H$, respectively. Drawn for $b<1$.

smaller than the Bragg angle $\theta_{\mathrm{B}}$ to the crystal surface. In the DuMond diagram of Fig. 2, the diffraction region is represented by band $O$ for incidence and by band $H$ for emergence, where the horizontal width of the former band is equal to $\omega_{\mathrm{s}} / \sqrt{b}$ and that of the latter to $\omega_{\mathrm{s}} \sqrt{ } b$. Because of the refraction effect, band $H$ is shifted from $O$ to the smaller glancing angle side.

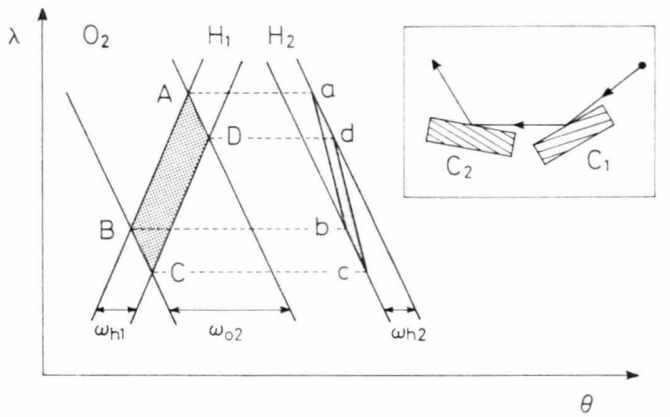

Fig. 3. DuMond diagram for double successive asymmetric diffractions in the $\left(n_{1},+n_{2}\right)$ arrangement. Band $O_{1}$ is not shown. An X-ray beam represented by abcd is obtained from crystal $C_{2}$. Drawn for $b_{1}<1$ and $b_{2}<1$.

Now we consider double successive asymmetric diffractions in the $\left(n_{1},+n_{2}\right)$ arrangement. We assume the asymmetry factors $b_{1}$ and $b_{2}$ of two crystals $\mathrm{C}_{1}$ and $\mathrm{C}_{2}$ to be smaller than unity (Fig. 3). The asymmetric diffraction used for the first crystal $\mathrm{C}_{1}$ selects the $\mathrm{X}$-rays represented by band $O_{1}$ from the incident beam and diffracts into $H_{1}$. These $\mathrm{X}$-rays impinge on the second crystal $\mathrm{C}_{2}$, by which those represented by region ABCD can be diffracted. Region ABCD is the overlapped region of bands $H_{1}$ and $\mathrm{O}_{2}$. Owing to the asymmetric diffraction in $\mathrm{C}_{2}$, a given wavelength component of these $\mathrm{X}$-rays is reduced in angular spread by a factor of $b_{2}$ when emerging from $\mathrm{C}_{2}$. Therefore $\mathrm{X}$-rays diffracted from $\mathrm{C}_{2}$ are represented by region abcd in Figure 3. It is to be noted that the spectral window of the X-rays obtained from the successive asymmetric diffractions is quite different from that obtained from the successive symmetric diffractions.

The $\lambda$-spread of the beam thus obtained is approximately given by

$$
\lambda_{0}\left(\omega_{\mathrm{s} 1} \sqrt{b_{1}}=\omega_{\mathrm{s} 2} / \sqrt{b_{2}}\right) /\left(\tan \theta_{\mathrm{B} 1}+\tan \theta_{\mathrm{B} 2}\right)
$$

where $\theta_{\mathrm{B} i}$ and $\omega_{\mathrm{S} i}$ are the Bragg angle and the angular range of total reflection of the $i$-th crystal for the symmetric diffraction, respectively, and $\lambda_{0}$ is the mean wavelength of the beam. Usually $\lambda_{0}$ is taken as the wavelength of the peak intensity of a characteristic line. The $\theta$-spread of the beam is given by

$$
\begin{aligned}
& \left(\omega_{\mathrm{s} 1} V b_{1}+\omega_{\mathrm{s} 2} / \sqrt{ } b_{2}\right) \tan \theta_{\mathrm{B} 2}^{\cdot}-\omega_{\mathrm{s} 2} / b_{\mathrm{s}} \\
& \tan \theta_{\mathrm{B} 1}+\tan \theta_{\mathrm{B} 2}
\end{aligned}
$$

for $b_{2} \leqq \tan \theta_{\mathrm{B} 2} /\left(\tan \theta_{\mathrm{B} 1}+\tan \theta_{\mathrm{B} 2}\right)$, and by

$$
\omega_{\mathrm{B} 2} \sqrt{ } b_{2}-\frac{\left(\omega_{\mathrm{s} 2} / \sqrt{b_{2}}-\omega_{\mathrm{s} 1} \sqrt{ } b_{1}\right) \tan \theta_{\mathrm{B} 2}}{\tan \theta_{\mathrm{B} 1}+\tan \theta_{\mathrm{B} 2}}
$$

for $b_{2}>\tan \theta_{\mathrm{B} 2} /\left(\tan \theta_{\mathrm{B} 1}+\tan \theta_{\mathrm{B} 2}\right)$. Equations (1) and (2) indicate that the $\theta$ - and $\lambda$-spreads of the beam are not very narrow in usual cases. However, when $b_{1}$ and $b_{2}$ are appropriately chosen, either of

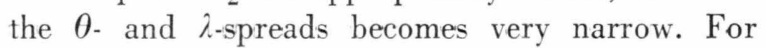
example, if

$$
b_{2}=\tan \theta_{\mathrm{B} 2} /\left(\tan \theta_{\mathrm{B} 1}+\tan \theta_{\mathrm{B} 2}\right),
$$

the $\theta$-spread is given by $\sqrt{b_{1}} b_{2} \omega_{\mathrm{s} 1}$, which is equal to the $\theta$-spread of a given wavelength component of the beam.

\subsection{Experiments}

In order to experimentally confirm the above investigations, rocking curves were measured in triplecrystal arrangements of $(n,+n, \pm n)$ settings, in

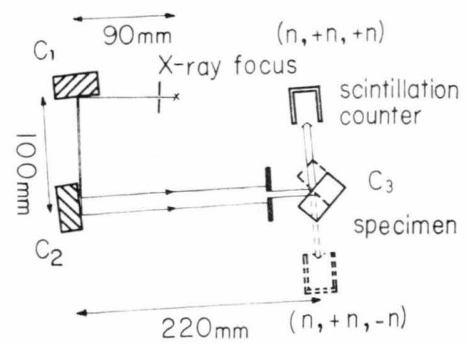

Fig. 4. Experimental disposition of three crystals $C_{1}, C_{2}$ and $\mathrm{C}_{3}$ in which rocking curves were measured for $(n,+n, \pm \mathrm{n})$ settings. 
which an asymmetric diffraction is used for the first and second crystals. Figure 4 shows the experimental disposition schematically. The three crystals $\mathrm{C}_{1}, \mathrm{C}_{2}$ and $\mathrm{C}_{3}$ were prepared from silicon single crystals of high purity and free from dislocations. The 422 diffraction of $\mathrm{CuK} \alpha_{1}$ was used. The asymmetry factors were chosen such that $b_{1}=$ $b_{2}=0.1$ and $b_{3}=1.0$. An ordinary X-ray tube was operated at $45 \mathrm{kV}$ and $20 \mathrm{~mA}$ with an apparent focal size of $0.5 \mathrm{~mm}$ in width and $1 \mathrm{~mm}$ in height. The beam incident on $\mathrm{C}_{3}$ was limited by a slit of $6 \mathrm{~mm}$ in width and $1 \mathrm{~mm}$ in height, with which the vertical divergence of the beam was limited within 8 min of arc.

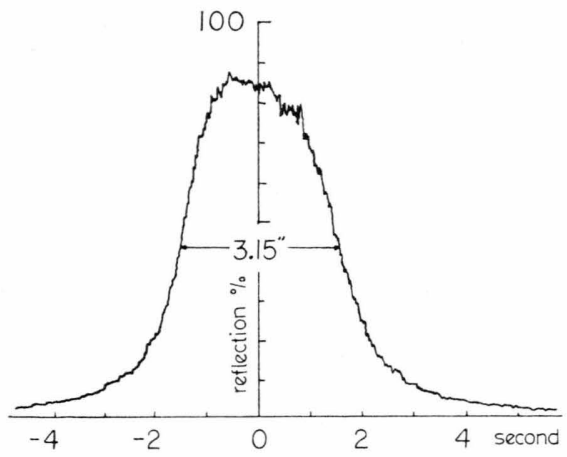

Fig. 5. A triple-crystal rocking curve observed for $(n,+n$, $-n$ ) setting of Figure 4 . Silicon 422 symmetric diffraction, $\mathrm{CuK} \alpha_{1}$.

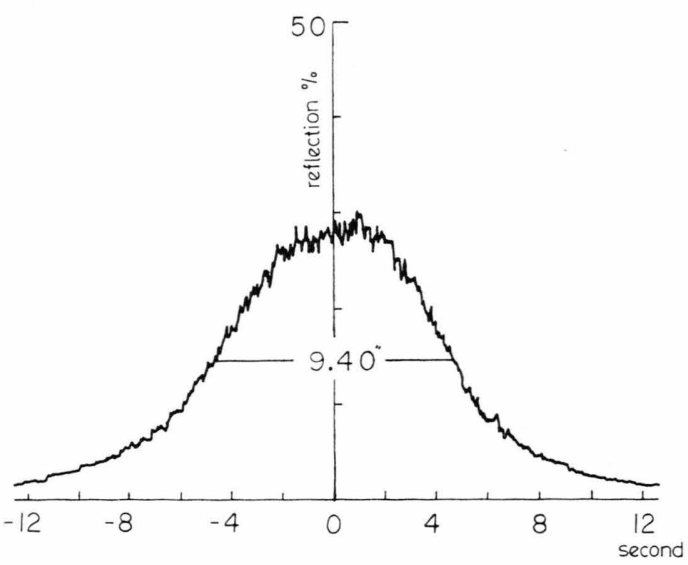

Fig. 6. A triple-crystal rocking curve observed for $(n,+n$, $+n$ ) setting of Figure 4 . Silicon 422 symmetric diffraction, $\mathrm{CuK} \alpha_{1}$.

Typical examples of the observed rocking curves are reproduced in Figs. 5 and 6. The half-value width of the curve observed for the $(n,+n,-n)$ setting was $3.15 \mathrm{sec}$, which is close to the intrinsic width $2.92 \mathrm{sec}$ of $\mathrm{C}_{3}$. The percent reflection was $85 \%$. For the $(n,+n,+n)$ setting the observed curve was $9.4 \mathrm{sec}$ in half-value width and $28.6 \%$ in percent reflection.

The profiles of the observed rocking curves are qualitatively explained in terms of the DuMond diagram as follows. In Figs. 7 and 8 the spectral window of the beam obtained from $\mathrm{C}_{1}$ and $\mathrm{C}_{2}$ is represented by abcd, and the diffraction region of $\mathrm{C}_{3}$ for incidence is shown by band $\mathrm{O}_{3}{ }^{-}$or $\mathrm{O}_{3}{ }^{+}$. When $\mathrm{C}_{3}$ is rotated about the vertical axis, band $\mathrm{O}_{3}{ }^{-}$or $\mathrm{O}_{3}{ }^{+}$translates in the horizontal direction in Figs. 7 and 8, and different regions of abcd are successively sampled. If we denote the intensity distribution of the beam abcd as $A_{0}$ and the reflection profile of $\mathrm{C}_{3}$ as $A_{\mathrm{s}}$, we have

$$
I(\gamma) \propto \iint A_{0}(\theta, \lambda) A_{\mathrm{s}}(\gamma-\theta, \lambda) \mathrm{d} \theta \mathrm{d} \lambda
$$

for the intensity diffracted from $\mathrm{C}_{3}$ for a given rotation angle $\gamma$ of $\mathrm{C}_{3}$. Putting

and

$$
B_{0}=\int A_{0}(\theta, \lambda) \mathrm{d} \lambda
$$

we obtain

$$
B_{\mathrm{s}}=\int A_{\mathrm{s}}(\gamma-\theta, \lambda) \mathrm{d} \lambda,
$$

$$
I(\gamma) \propto \int B_{0}(\theta) B_{\mathrm{s}}(\gamma-\theta) \mathrm{d} \theta .
$$

$B_{0}$ and $B_{\mathrm{S}}$ represent the intensity profile of the exploring beam and the reflection profile of $\mathrm{C}_{3}$ integrated over the wavelength, respectively, and are shown schematically in the middles of Figs. 7 and 8. Now the rocking curves to be observed are given by convolutions of $B_{0}$ and $B_{\mathrm{s}}$ as shown schematically in the bottoms of Figs. 7 and 8. If we assume that both $B_{0}$ and $B_{\mathrm{s}}$ are expressed as Gaussians, the

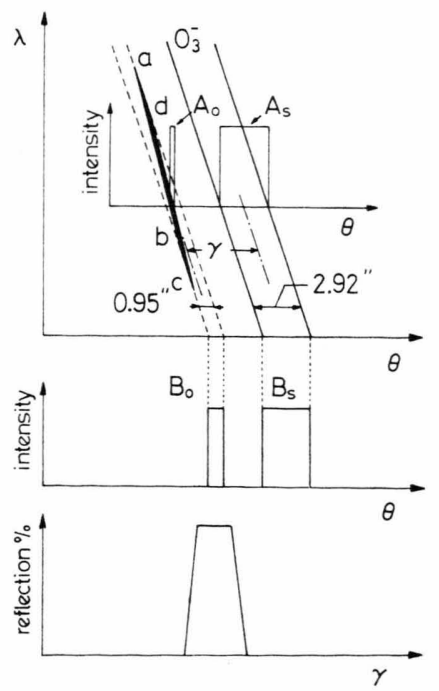

Fig. 7. Explaining the profile of the rocking curve observed for $(n,+n,-n)$ setting of Figure 4. 


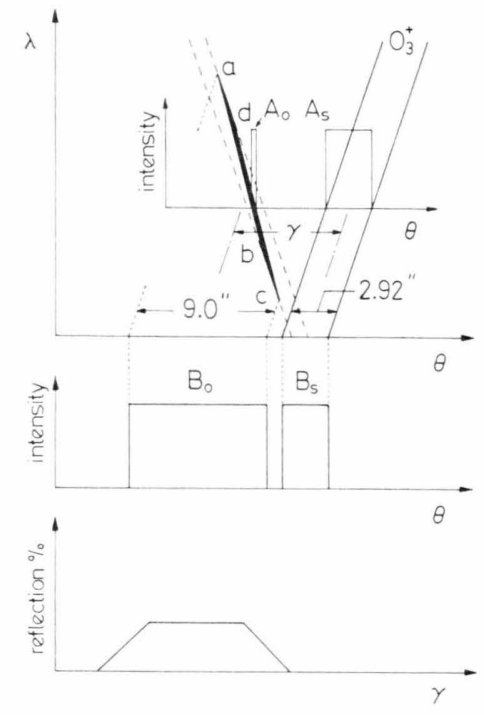

Fig. 8. Explaining the profile of the rocking curve observed for $(n,+n,+n)$ setting of Figure 4.

half-value widths of the rocking curves are given as $\sqrt{0.95^{2}+2.92^{2}}=3.07 \mathrm{sec}$ for the $(n,+n,-n)$ setting and $\sqrt{ } 9.0^{2}+0.95^{2}=9.5 \mathrm{sec}$ for the $(n,+n$, $+n$ ) setting. These values are in a good agreement with the observations. A more quantitative analysis will be given elsewhere in the near future.

\section{A Method of Obtaining a Highly Parallel and Monochromatic Beam by Triple Successive Asymmetric Diffractions}

\subsection{Principle}

As can be seen from Eqs. (1) and (2), double successive asymmetric diffractions do not usually produce an X-ray beam whose $\theta$ - and $\lambda$-spreads are very narrow at the same time. Here we describe a method for obtaining a highly parallel and monochromatic beam with the use of triple successive asymmetric diffractions. Figure 9 shows the prin-

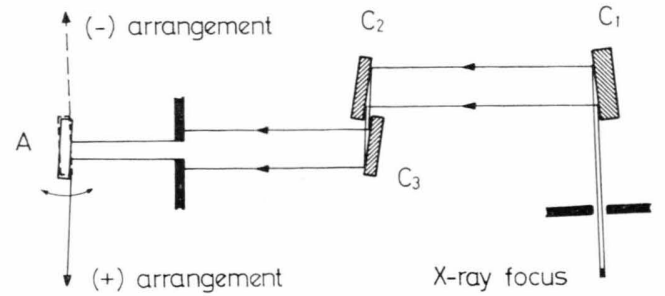

Fig. 9. Showing arrangement of three crystals $C_{1}, C_{2}$ and $C_{3}$ for obtaining a highly parallel and monochromatic beam with the use of triple successive asymmetric diffractions. An ana-

lyser crystal (A) is used for studying the obtained beam. ciple schematically. Three crystals $\mathrm{C}_{1}, \mathrm{C}_{2}$, and $\mathrm{C}_{3}$ are arranged in the $(n,+n,-n)$ setting, for each of which an asymmetric diffraction takes place. The asymmetry factors of the three crystals, $b_{1}, b_{2}$ and $b_{3}$, are chosen such that $b_{1}=1 / b_{2}=b_{3}<1$. In the DuMond diagram of Fig. 10, X-rays diffracted from $\mathrm{C}_{1}$ are represented by band $H_{1}$ of an angular or horizontal width of $\sqrt{ } b_{1} \omega_{\mathrm{s}}$. Of these X-rays those represented by $\mathrm{ABCD}$ are selected by $\mathrm{C}_{2}$. Because the asymmetry factor $b_{2}$ is larger than unity, the horizontal width of band $\mathrm{O}_{2}$ is reduced to $\omega_{\mathrm{s}} / \mathrm{V} b_{2}$ and therefore the window $A B C D$ is very small in

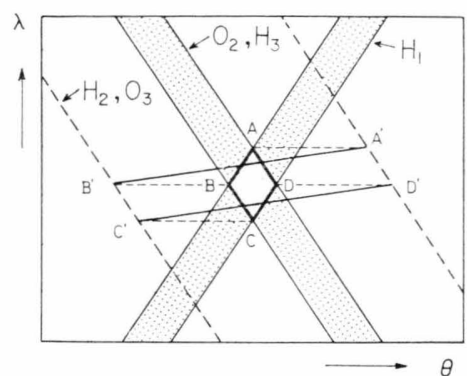

Fig. 10. Explaining the principle of the method shown in Fig. 9 in terms of the DuMond diagram. Horizontal shift of $\mathrm{H}_{2}$ and $\mathrm{O}_{3}$ from $\mathrm{O}_{2}$ and $\mathrm{H}_{3}$ is not shown for brevity. An X-ray beam represented by $\mathrm{ABCD}$ is obtained.

both of the $\theta$ - and $\lambda$-spreads. However, the same asymmetry of $\mathrm{C}_{2}$ expands the $\theta$-spread by a factor of $b_{2}$ for emergence, so that the $\mathrm{X}$-rays diffracted from $\mathrm{C}_{2}$ are given by $\mathrm{A}^{\prime} \mathrm{B}^{\prime} \mathrm{C}^{\prime} \mathrm{D}^{\prime}$. Because the asymmetry factor $b_{3}$ of $\mathrm{C}_{3}$ is reciprocal to $b_{2}$, the whole of the $\mathrm{X}$-rays are further diffracted by $\mathrm{C}_{3}$ and leaves $\mathrm{C}_{3}$ with a spectral window represented again by $\mathrm{ABCD}$. The $\mathrm{X}$-ray beam thus obtained is $\sqrt{ } b_{1} \omega_{\mathrm{s}}$ in $\theta$-spread and $\lambda_{0} \sqrt{b_{1}} \omega_{\mathrm{s}} / \tan \theta_{\mathrm{B}}$ in $\lambda$-spread.

\subsection{Experiments}

A monochro-collimator system was designed which consisted of three silicon crystals with $b_{1}=$ $1 / b_{2}=b_{3}=0.1$ for asymmetric 422 diffraction of $\mathrm{CuK} \alpha_{1}$. All the crystal components were prepared from a highly perfect crystal of silicon. The second and third components were parts of a monolithic block separated from each other by a parallel groove milled away. The calculated $\theta$ - and $\lambda$-spreads of the $\mathrm{X}$-rays to be obtained from the system are approximately $1.0 \mathrm{sec}$ of arc and $7.7 \times 10^{-6} \AA$, i. e. $\Delta \lambda / \lambda_{0}$ $=5.0 \times 10^{-6}\left(\lambda_{0}=1.54 \AA\right)$, respectively.

The performance of the monochro-collimator was investigated by measuring rocking curves with a silicon analyzer crystal, which uses an asymmetric 
422 diffraction of $b=33$ (Figure 9). The angular resolution of this analyzer is about $0.6 \mathrm{sec}$. An Xray tube of an apparent focal size of $0.8 \mathrm{~mm}$ in width and $0.4 \mathrm{~mm}$ in height was operated at $40 \mathrm{kV}$ and $20 \mathrm{~mA}$.

The half-value widths of the observed curves were 0.96 and $1.17 \mathrm{sec}$ for the $(+)$ and $(-)$ settings of the analyzer, respectively. These values agree well with $1.17 \mathrm{sec}$ which we obtain by assuming simply both the angular distribution of the exploring beam and the intrinsic diffraction curve of the analyzer crystal to be Gaussian. The narrower half-value width is to be expected for the $(+)$ setting of the analyzer, because the exploring beam is more sharply cut in tails on the sides $\mathrm{AB}$ and $\mathrm{CD}$ than on $\mathrm{AD}$ and $\mathrm{BC}$ of the window $\mathrm{ABCD}$ of Fig. 10 owing to the successive diffractions used in the parallel groove ${ }^{23}$. The intensity of the X-ray beam as currently obtained amounted to 170 counts/ $\mathrm{sec} / \mathrm{mm}^{2}$ when measured at a distance of $42 \mathrm{~cm}$ from the X-ray focus.

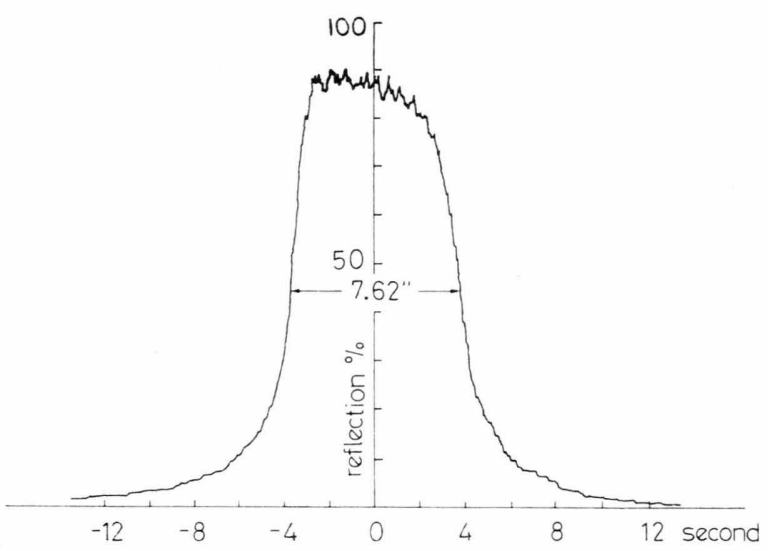

Fig. 11. Rocking curve observed from a silicon crystal for the symmetric 111 diffraction with $\mathrm{CuK} \alpha_{1}$. The beam obtained with the method of Fig. 9 was used as the exploring beam.

It is worthwhile noticing that the X-rays presently obtained are almost perfectly polarized because twice the Bragg angle of silicon 422 diffraction with $\mathrm{CuK} \alpha_{1}$ is nearly equal to $90^{\circ}$, so that the intensity ratio of the $\pi$ to the $\sigma$ components of polarization is smaller than $2 \times 10^{-5}$.

An example demonstrating the potential utility of the obtained beam is given in Fig. 11, which reproduces a rocking curve observed from a silicon crystal for the symmetric 111 diffraction. The specimen was set in a position corresponding to the $(+)$ arrangement of Figure 11. The observed curve reveals the flat-topped asymmetric profile of the dynamical diffraction curve, which is expected from the theory ${ }^{24}$ assuming an ideally parallel and monochromatic incident beam. The half-value width of the observed curve is $7.62 \mathrm{sec}$ which is to be compared with the intrinsic width $7.39 \mathrm{sec}$ (calculated from the Hartree-Fock scattering factor for the free atom) of the specimen for the $\sigma$ component of polarization.

\section{Discussion}

(i) Investigations made in Sect. 2 show that an X-ray beam of a peculiar spectral window can be obtained from double successive asymmetric diffractions in the $\left(n_{1},+n_{2}\right)$ arrangement. Various shapes of the spectral window are realized with combinations of $\theta_{\mathrm{B}}, \omega_{\mathrm{s}}$ and $b$. In particular, if $b_{2}$ is chosen so as to satisfy Eq. (3), the spectral window becomes vertical in the DuMond diagram. In other words, the beam diffracted from the second crystal is highly parallel not only for a given wavelength but also for an extended range of wavelengths, although it is not highly monochromatic. Such a condition would be useful, for example, for the diffraction topography utilizing double-crystal arrangements ${ }^{25}$ : Even when the photographic plate is placed rather distant from the second or specimen crystal, the topographs to be obtained would not be impaired in spatial resolution. It is also possible to obtain a beam very narrow in $\theta$-spread but rather wide in $\lambda$-spread. This can be achieved, e. g., when $b_{1}<1$ and $b_{2}>1$. A more detailed analysis of the spectral window is now being made.

(ii) As mentioned before, a highly parallel and monochromatic beam can be obtained also with double higher-order symmetric diffractions in the $\left(n_{1},+n_{2}\right)$ arrangement. However, for given $\theta$ - and $\lambda$-spreads of the beam, the method described in Sect. 3 gives a total beam intensity stronger by approximately a factor of $1 / b_{1}$. This is because, in the method of Sect. 3 , the first crystal $C_{1}$ can diffract X-rays of angular spread approximately $1 / b_{1}$ times wider than the $\theta$-spread of the finally obtained beam, while, in the method using higher-order symmetric diffractions, the angular range of diffraction for incidence of the first crystal is approximately the same as the $\theta$-spread of the resultant beam. It is to be noted that the beam obtained with the method of Sect. 3 possesses a horizontal width $1 / b_{1}$ times wider than that of the X-ray focus used. According$\mathrm{ly}$, for a given focus of the $\mathrm{X}$-ray source, the two methods give approximately same intensity per unit 
horizontal width of the beam, although the total intensity is $1 / b_{1}$ times stronger for the method of Sect. 3 as noted above.

(iii) It is possible to obtain an X-ray beam of parallelism and monochromacy higher than that obtained in Sect. 3.2 by appropriately choosing the values of $b_{1}$ and $b_{2}$. In order to overcome a reduction in intensity (per unit horizontal width) a fine-focus X-ray source of high brilliancy could favourably be used.

(iv) In the method of Sect. 3 an asymmetry factor less than unity $\left(b_{1}<1\right)$ is used for the first crystal $\mathrm{C}_{1}$. It is also possible to obtain a highly parallel and monochromatic beam with $b_{1}>1$. In this case, we must use, at least, quadruple successive asymmetric diffractions as shown in Fig. 12, where four crystals $\mathrm{C}_{1}, \mathrm{C}_{2}, \mathrm{C}_{3}$ and $\mathrm{C}_{4}$ of $b_{1}=1 / b_{2}=b_{3}=$ $1 / b_{4}>1$ are arranged in the $(n,-n,+n,-n)$ setting. The system is easily prepared by grooving two blocks of single crystals. The beam thus obtained, however, is weaker in total intensity than that obtained with the method of Sect. 3: For a given spread in $\theta$ and $\lambda$ of the beam, the total inten-

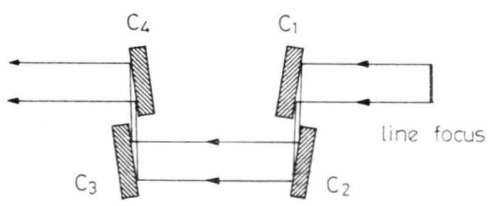

Fig. 12. Quadruple successive asymmetric diffractions for obtaining a highly parallel and monochromatic X-ray beam.

1 G. Borrmann and K. Kohra, Read before Meeting of Phys. Soc. Japan [1958, April].

2 A. Authier, C. R. Acad. Sci. Paris 251, 2003 [1960].

3 A. Authier, C. R. Acad. Sci. Paris 251, 2502 [1960].

4 A. Authier, Bull. Soc. Fr. Miner. 84, 51 [1961].

5 S. Nakano, Dr. Thesis presented to Fac. of Sci., Univ. of Tokyo 1965.

6 M. Renninger, Z. Naturforsch. 16 a, 1110 [1961].

7 K. Kohra, J. Phys. Soc. Japan 17, 589 [1962].

8 K. Kohra and S. Kikuta, Acta Cryst. A 24, 200 [1968].

9 S. Kikuta and K. Kohra, J. Phys. Soc. Japan 29, 1322 [1970].

10 S. Kikuta, J. Phys. Soc. Japan 30, 222 [1971].

11 T. Matsushita, S. Kikuta, and K. Kohra, J. Phys. Soc. Japan 30, 1136 [1971].

12 S. Kikuta and K. Kohra, J. Phys. Soc. Japan 25, 924 [1968].

13 H. Hashizume, K. Nakayama, T. Matsushita, and K. Kohra, J. Phys. Soc. Japan 29, 806 [1970]. sity obtained by using quadruple asymmetric diffractions is weaker by a factor of $1 / b_{1}{ }^{q}\left(=1 / b_{1}{ }^{t}\right)$ for the same reason as given in (ii), where superscripts $\mathrm{t}$ and $\mathrm{q}$ mean the triple and quadruple successive diffractions, respectively. The system shown in Fig. 12 would be useful when an X-ray source of a line focus is available.

An advantage taken from the quadruple successive diffration is that the obtained beam has a spectral window of which both pairs of sides are equally sharply cut, in contrast to those obtained with the method of Sect.3. However, sharply cut sides of the spectral window can be realized also with the method of Sect. 3 by using two additional asymmetric diffractions in front of $\mathrm{C}_{1}$ : Fivefold successive asymmetric diffractions in the $(n,-n$, $+n,+n,-n)$ arrangement with $b_{1}=1 / b_{2}=b_{3}=$ $1 / b_{4}=b_{5}<1$.

(v) It is convenient to classify monochro-collimator systems utilizing multiple asymmetric diffractions into two types according to whether the asymmetry factor used for the first crystal is smaller or greater than unity. The simplest versions of the two types are represented in Figs. 9 and 12. It is possible to add pairs of asymmetric diffractions in front of the first crystal and/or behind the last crystal of the arrangements of Figs. 9 and 12. The addition does not essentially change the $\theta$ - and $\lambda$-spreads of the obtained beam nor its total intensity, but only sharpens the sides of the spectral window.

14 S. Kikuta, T. Matsushita, and K. Kohra, Phys. Letters 33 A, 151 [1971].

15 S. Kikuta, Phys. Stat. Sol. (b) 45, 333 [1971].

16 S. Kikuta and K. Kohra, J. Phys. Soc. Japan 25, 923 [1968].

17 K. Nakayama, H. Hashizume, and K. Kohra, J. Phys. Soc. Japan 30, 893 [1971].

18 M. Renninger, Naturwiss. 40, 50 [1953].

19 M. Renninger, Acta Cryst. 8, 597 [1955].

20 M. Bubakova, J. Drahokoupil, and A. Fingerland, Czech. J. Phys. B 11, 199 [1961].

21 R. Bubakova, J. Drahokoupil, and A. Fingerland, Czech. J. Phys. B 12, 764 [1962].

22 J. W. M. DuMond, Phys. Rev. 52, 872 [1937].

23 U. Bonse and M. Hart, Appl. Phys. Letters 7, 238 [1965].

24 M. von Laue, Röntgenstrahlinterferenzen, Akademische Verlagsgesellschaft, Frankfurt am Main, 1960.

25 K. Kohra, H. Hashizume, and J. Yoshimura, Japan. J. Appl. Phys. 9, 1029 [1970]. 